\title{
Time of Flight
}

National Cancer Institute

\section{Source}

National Cancer Institute. Time of Flight. NCI Thesaurus. Code C111985.

Estimation of the difference in annihilation photon travel time from emission to detection within the imaging device to help localize the site of positron emission. 